\title{
Hidden Symmetries of Stochastic Models ${ }^{\star}$
}

\section{Boyka ANEVA}

Institute for Nuclear Research and Nuclear Energy, Bulgarian Academy of Sciences, 72 Tsarigradsko chaussee, 1784 Sof ia, Bulgaria

E-mail:blan@inrne.bas.bg

Received November 23, 2006, in final form May 04, 2007; Published online May 18, 2007

Original article is available at http://www.emis.de/journals/SIGMA/2007/068/

\begin{abstract}
In the matrix product states approach to $n$ species diffusion processes the stationary probability distribution is expressed as a matrix product state with respect to a quadratic algebra determined by the dynamics of the process. The quadratic algebra defines a noncommutative space with a $S U_{q}(n)$ quantum group action as its symmetry. Boundary processes amount to the appearance of parameter dependent linear terms in the algebraic relations and lead to a reduction of the $S U_{q}(n)$ symmetry. We argue that the boundary operators of the asymmetric simple exclusion process generate a tridiagonal algebra whose irriducible representations are expressed in terms of the Askey-Wilson polynomials. The Askey-Wilson algebra arises as a symmetry of the boundary problem and allows to solve the model exactly.
\end{abstract}

Key words: stohastic models; tridiagonal algebra; Askey-Wilson polynomials

2000 Mathematics Subject Classification: 60J60; 17B80

\section{Introduction}

Stochastic reaction-diffusion processes are of both theoretical and experimental interest not only because they describe various mechanisms in physics and chemistry [1] but they also provide a way of modelling phenomena like traffic flow [2], kinetics of biopolimerization [3], interface growth [4].

A Markov process can be described in terms of a master equation for the probability distribution $P\left(s_{i}, t\right)$ of a stochastic variable $s_{i}=0,1,2, \ldots, n-1$ at a site $i=1,2, \ldots, L$ of a linear chain. A state on the lattice at a time $t$ is determined by the occupation numbers $s_{i}$ and a transition to another configuration $s_{i}^{\prime}$ during an infinitesimal time step $d t$ is given by the probability $\Gamma\left(s, s^{\prime}\right) d t$. The rates $\Gamma \equiv \Gamma_{j l}^{i k}, i, j, k, l=0,1,2, \ldots, n-1$ are assumed to be independent from the position in the bulk. At the boundaries, i.e. sites 1 and $L$ additional processes can take place with rates $L_{i}^{j}$ and $R_{i}^{j}$. Due to probability conservation

$$
\Gamma(s, s)=-\sum_{s^{\prime} \neq s} \Gamma\left(s^{\prime}, s\right) .
$$

The master equation for the time evolution of a stochastic system

$$
\frac{d P(s, t)}{d t}=\sum_{s^{\prime}} \Gamma\left(s, s^{\prime}\right) P\left(s^{\prime}, t\right)
$$

is mapped to a Schrödinger equation for a quantum Hamiltonian in imaginary time

$$
\frac{d P(t)}{d t}=-H P(t)
$$

${ }^{\star}$ This paper is a contribution to the Proceedings of the O'Raifeartaigh Symposium on Non-Perturbative and Symmetry Methods in Field Theory (June 22-24, 2006, Budapest, Hungary). The full collection is available at http://www.emis.de/journals/SIGMA/LOR2006.html 
where

$$
H=\sum_{j} H_{j, j+1}+H^{(L)}+H^{(R)}
$$

The probability distribution thus becomes a state vector in the configuration space of the quantum spin chain and the ground state of the Hamiltonian, in general non-Hermitian, corresponds to the steady state of the stochastic dynamics where all probabilities are stationary. The mapping provides a connection with integrable quantum spin chains and allows for exact results of the stochastic dynamics with the formalism of quantum mechanics.

Examples are the diffusion processes of particles hopping with hard core repulsion between lattice sites $i, j$ with probability rates $g_{i j}$ and subjected to reflecting (or free) boundary conditions [5]. In the symmetric case $g_{i j}=g_{j i}$ the stochastic Hamiltonian is the $S U(2)$ symmetric spin $1 / 2$ isotropic Heisenberg ferromagnet

$$
H=-\frac{1}{2} \sum_{i}\left(\sigma_{i}^{x} \sigma_{j}^{x}+\sigma_{i}^{y} \sigma_{j}^{y}+\sigma_{i}^{z} \sigma_{j}^{z}-1\right) .
$$

The $S U(2)$ symmetry, yet unrevealed in the original master equation becomes manifest through the mapping and allows for exact results of the stochastic dynamics. The asymmetric exclusion process is the driven diffusive lattice gas of particles hopping with rates $\frac{g_{i, i+1}}{g_{i+1, i}}=q \neq 1$ and is mapped to a $S U_{q}(2)$-symmetric $X X Z$ chain with anisotropy $\Delta=\frac{\left(q+q^{-1}\right)}{2}$.

\section{Matrix product state approach to diffusion models}

An algebraic approach, developed for the study of the steady state properties of Markov processes and related to the quantum Hamiltonian picture is the matrix-product-state ansatz [6, 7]. The idea is that the stationary probability distribution, i.e. the ground state of the quantum Hamiltonian with nearest-neighbour interaction in the bulk and single-site boundary terms is expressed as a product of (or a trace over) matrices that form a representation of a quadratic algebra

$$
\Gamma_{j l}^{i k} D_{i} D_{k}=x_{l} D_{j}-x_{j} D_{l}, \quad i, j, k=0,1, \ldots, n-1,
$$

determined by the dynamics of the process.

In the following we consider diffusion processes with $n$ species on a chain of $L$ sites with nearest-neighbour interaction with exclusion, i.e. a site can be either empty or occupied by a particle of a given type. In the set of occupation numbers $\left(s_{1}, s_{2}, \ldots, s_{L}\right)$ specifying a configuration of the system $s_{i}=0$ if a site $i$ is empty, $s_{i}=1$ if there is a first-type particle at a site $i, \ldots, s_{i}=n-1$ if there is an $(n-1)$ th-type particle at a site $i$. On successive sites the species $i$ and $k$ exchange places with probability $g_{i k} d t$, where $i, k=0,1,2, \ldots, n-1$. With $i<k, g_{i k}$ are the probability rates of hopping to the left, and $g_{k i}$ to the right. The event of exchange happens if out of two adjacent sites one is a vacancy and the other is occupied by a particle, or each of the sites is occupied by a particle of a different type. The $n$-species symmetric simple exclusion process is known as the lattice gas model of particle hopping between nearest-neighbour sites with a constant rate $g_{i k}=g_{k i}=g$. The $n$-species asymmetric simple exclusion process with hopping in a preferred direction is the driven diffusive lattice gas of particles moving under the action of an external field. The process is totally asymmetric if all jumps occur in one direction only, and partially asymmetric if there is a different non-zero probability of both left and right hopping. The number of particles $n_{i}$ of each species in the bulk is conserved $\sum_{i=0}^{n-1} n_{i}=L$ and this 
is the case of periodic boundary conditions. In the case of open systems, the lattice gas is coupled to external reservoirs of particles of fixed density. In most studied examples $[7,8]$ one considers phase transitions inducing [9] boundary processes when a particle of type $k, k=1,2, \ldots, n-1$ is added with a rate $L_{k}^{0}$ and/or removed with a rate $L_{0}^{k}$ at the left end of the chain, and it is removed with a rate $R_{0}^{k}$ and/or added with a rate $R_{k}^{0}$ at the right end of the chain.

For diffusion processes the transition rate matrix becomes simply $\Gamma_{k i}^{i k}=g_{i k}$ and the $n$-species diffusion algebra [10] has the form

$$
g_{i k} D_{i} D_{k}-g_{k i} D_{k} D_{i}=x_{k} D_{i}-x_{i} D_{k}
$$

where $g_{i k}$ and $g_{k i}$ are positive (or zero) probability rates, $i, k=0,1, \ldots, n-1$ and $x_{i}$ satisfy

$$
\sum_{i=0}^{n-1} x_{i}=0 .
$$

(No summation over repeated indices in equation (2.1).)

The quadratic algebra has a Fock representation in an auxiliary Hilbert space where the $n$ generators act as operators. For systems with periodic boundary conditions, the stationary probability distribution is related to the expression

$$
P\left(s_{1}, \ldots, s_{L}\right)=\operatorname{Tr}\left(D_{s_{1}} D_{s_{2}} \cdots D_{s_{L}}\right) .
$$

When boundary processes are considered the stationary probability distribution is related to a matrix element in the auxiliary vector space

$$
P\left(s_{1}, \ldots, s_{L}\right)=\left\langle w\left|D_{s_{1}} D_{s_{2}} \cdots D_{s_{L}}\right| v\right\rangle
$$

with respect to the vectors $|v\rangle$ and $\langle w|$, defined by the boundary conditions

$$
\left\langle w\left|\left(L_{i}^{k} D_{k}+x_{i}\right)=0, \quad\left(R_{i}^{k} D_{k}-x_{i}\right)\right| v\right\rangle=0,
$$

where the $x$ sum up to zero, because of the form of the boundary rate matrices

$$
L_{i}^{i}=-\sum_{j=0}^{L-1} L_{j}^{i}, \quad R_{i}^{i}=-\sum_{j=0}^{L-1} R_{j}^{i} .
$$

These relations simply mean that one associates with an occupation number $s_{i}$ at position $i$ a matrix $D_{s_{i}}=D_{k}(i=1,2, \ldots, L ; k=0,1, \ldots, n-1)$ if a site $i$ is occupied by a $k$-type particle. The number of all possible configurations of an $n$-species stochastic system on a chain of $L$ sites is $n^{L}$ and this is the dimension in the configuration space of the stationary probability distribution as a state vector. Each component of this vector, i.e. the (unnormalized) steadystate weight of a given configuration, is a trace or an expectation value in the auxiliary space given by (2.2) or (2.3). The quadratic algebra reduces the number of independent components to only monomials symmetrized upon using the relations (2.1).

The algebra admits an involution, hence the matrices $D_{i}$ can be chosen Hermitian

$$
D_{i}=D_{i}^{+}, \quad g_{i k}^{+}=g_{k i}, \quad x_{i}=-x_{i}^{+}
$$

(or $D_{i}=-D_{i}^{+}$, if $g_{i k}=g_{k i}^{+}$).

The relations (2.1) allow an ordering of the elements $D_{k}$. Monomials of given order are the Poincaré-Birkhoff-Witt (PBW) basis in the algebra. The quadratic algebra generated by the $n$ 
elements $D_{k}$ obeying the $n(n-1) / 2$ relations $(2.1)$ is an associative algebra with a unit $e$. The ordered monomials

$$
D_{s_{1}}^{n_{1}} D_{s_{2}}^{n_{2}} \cdots D_{s_{l}}^{n_{l}},
$$

where $s_{1}<s_{2}<\cdots<s_{l}, l \geq 1$ and $n_{1}, n_{2}, \ldots, n_{l}$ are non-negative integers, are a linear basis in the algebra.

Thus, to find the stationary probability distribution one has to compute traces or matrix elements with respect to the vectors $|v\rangle$ and $\langle w|$ of monomials of the form $D_{s_{1}}^{n_{1}} D_{s_{2}}^{n_{2}} \cdots D_{s_{L}}^{n_{L}}$. The problem to be solved is twofold - to find a representation of the matrices $D$ that is a solution of the quadratic algebra and match the algebraic solution with the boundary conditions. Finding a representation of the quadratic algebra and solving the corresponding boundary problem allows one to conclude about the symmetries of the model.

In the case of symmetric diffusion with all $x_{i}$ distinct the diffusion algebra is of Lie-algebra type. The $n$ generators $D_{i}$, and $e$ can be mapped [11] to the generators $J_{j k}$ of $S U(n) \times U(1)$ and the mapping is invertible. The universal enveloping algebra generated by $D_{i}$ belongs to the UEA of the Lie-algebra of $S U(n) \times U(1)$.

In the known example of exactly solvable 2 - and 3 -species models $[7,12,13]$ of asymmetric diffusion through the matrix product ansatz, the solution of the quadratic algebra is provided by a deformed bosonic oscillator algebra, if both $g_{i k}$ and $g_{k i}$ differ from zero, or by infinitedimensional matrices, if $g_{i k}=0$. In the general $n$ case, because of the ordering procedure, the solution of the quadratic algebra has to be consistent with the diamond lemma in ring theory, also known as the braid associativity condition in quantum groups. As shown in [10, 11] if all parameters $x_{i}$ on the RHS of equation (2.1) are equal to zero, the homogeneous quadratic algebra defines a multiparameter quantized non-commutative space realized equivalently as a $q$-deformed Heisenberg algebra [14, 15] of $n$ oscillators depending on $n(n-1) / 2+1$ parameters (in general on $n(n-1) / 2+n$ parameters):

$$
\begin{array}{ll}
a_{i} a_{i}^{+}-r_{i} a_{i}^{+} a_{i}=1, & a_{i}^{+} a_{j}^{+}-q_{j i} a_{j}^{+} a_{i}^{+}=0, \\
a_{i} a_{j}-q_{j i} a_{j} a_{i}=0, & a_{i} a_{j}^{+}-q_{j i}^{-1} a_{j}^{+} a_{i}=0,
\end{array}
$$

where $i<j ; i, j=0,1, \ldots, n-1$, the deformation parameters $r_{i}, q_{i j}$ are model-dependent parameters given in terms of the probability rates. The associative algebra generated by the elements $D_{i}$ in this case belongs to the universal enveloping algebra of the multiparameter deformed Heisenberg algebra to which a consistent multiparameter $S U_{q}(n)$ quantization corresponds. Thus the bulk symmetry of a process without boundary conditions is $S U_{q}(n)$ and the process is equivalent to an integrable spin chain. For a non-homogeneous algebra with $x$ terms on the RHS of (2.1), only then is braid associativity satisfied if, out of the coefficients $x_{i}, x_{k}, x_{l}$ corresponding to an ordered triple $D_{i} D_{k} D_{l}$, either one coefficient $x$ is zero or two coefficients $x$ are zero, and the rates are respectively related. The diffusion algebras in this case can be obtained by either a change of basis in the $n$-dimensional non-commutative space or by a suitable change of basis of the lower-dimensional quantum space realized equivalently as a lower-dimensional deformed Heisenberg algebra. The appearance of the non-zero linear terms in the RHS of the quantum plane relations due to boundary processes leads to a lowerdimensional non-commutative space and a reduction of the $S U_{q}(n)$ invariance.

\section{The open asymmetric exclusion process}

We consider now the two-species partially asymmetric simple exclusion process with incoming and outgoing particles at both boundaries. We simplify the notations, namely, at the left boundary a particle can be added with probability $\alpha d t$ and removed with probability $\gamma d t$, and at the 
right boundary it can be removed with probability $\beta d t$ and added with probability $\delta d t$. The system is described by the configuration set $s_{1}, s_{2}, \ldots, s_{L}$ where $s_{i}=0$ if a site $i=1,2, \ldots, L$ is empty and $s_{i}=1$ if a site $i$ is occupied by a particle. The particles hop with a probability $g_{01} d t$ to the left and with a probability $g_{10} d t$ to the right, where without loss of generality we can choose the right probability rate $g_{10}=1$ and the left probability rate $g_{01}=q$. The model depends on five parameters - the bulk probability rate $q$ and the four boundary rates. The totally asymmetric process corresponds to $q=0$. The quadratic algebra

$$
D_{1} D_{0}-q D_{0} D_{1}=x_{1} D_{0}-D_{1} x_{0}, \quad x_{0}+x_{1}=0
$$

is solved [16] by a pair of deformed oscillators $a, a^{+}\left(\right.$and $\left.x_{0}=-x_{1}=1\right)$

$$
D_{0}=\frac{1}{1-q}+\frac{a^{+}}{\sqrt{1-q}}, \quad D_{1}=\frac{1}{1-q}+\frac{a}{\sqrt{1-q}} .
$$

The boundary conditions have the form

$$
\left(\beta D_{1}-\delta D_{0}\right)|v\rangle=|v\rangle, \quad\langle w|\left(\alpha D_{0}-\gamma D_{1}\right)=\langle w| .
$$

For a given configuration $\left(s_{1}, s_{2}, \ldots, s_{L}\right)$ the stationary probability is given by the expectation value

$$
P(s)=\frac{\left\langle w\left|D_{s_{1}} D_{s_{2}} \cdots D_{s_{L}}\right| v\right\rangle}{Z_{L}}
$$

where $D_{s_{i}}=D_{1}$ if a site $i=1,2, \ldots, L$ is occupied and $D_{s_{i}}=D_{0}$ if a site $i$ is empty and $Z_{L}=\left\langle w\left|\left(D_{0}+D_{1}\right)^{L}\right| v\right\rangle$ is the normalization factor to the stationary probability distribution. Once the representation of the diffusion algebra and the boundary vectors are known, one can evaluate all the physical quantities such as the current $J$ through a bond between site $i$ and site $i+1$, which has a very simple form

$$
J=\frac{Z_{L-1}}{Z_{L}}
$$

the mean density at a site $i$

$$
\left\langle s_{i}\right\rangle=\frac{\left\langle w\left|\left(D_{0}+D_{1}\right)^{i-1} D_{1}\left(D_{0}+D_{1}\right)^{L-i}\right| v\right\rangle}{Z_{L}},
$$

the two-point correlation function

$$
\left\langle s_{i} s_{j}\right\rangle=\frac{\left\langle w\left|\left(D_{0}+D_{1}\right)^{i-1} D_{1}\left(D_{0}+D_{1}\right)^{j-i-1} D_{1}\left(D_{0}+D_{1}\right)^{L-j}\right| v\right\rangle}{Z_{L}}
$$

and higher correlation functions.

The matrix-product algebraic relations provide solvable (and representation independent) recursions for the stationary probability, the current and the correlation functions. These recursions have been obtained in earlier works $[8,17]$, however they were not readily generalized to other models. The algebraic matrix-product method allows for generalizations to the multispecies case [7] and was also to the dynamical process [18].

To solve the boundary problem for a process with only incoming particles at the left boundary and only outgoing particles at the right one $(\delta=\gamma=0$ in (3.1)) we choose the vector $|v\rangle$ to be the (unnormalized!) eigenvector of the annihilation operator $a$ for a real value of the parameter $v$ and the vector $\langle w|$ to be the eigenvector (unnormalized and different from the conjugated one) of the creation operator for the real parameter $w$ :

$$
|v\rangle=e_{q}^{-\frac{1}{2} v w} e_{q}^{v a^{+}}|0\rangle, \quad\langle w|=\langle 0| e_{q}^{w a} e_{q}^{-\frac{1}{2} w v},
$$


where $e_{q}^{z}=\sum \frac{z^{n}}{[n] !}$ and $[n]=\frac{1-q^{n}}{1-q}$. The factor $e_{q}^{-\frac{1}{2} v w}$ in (3.2) is due to the condition $\langle w \mid v\rangle=1$, which is a convenient choice in physical applications. According to the algebraic solution, these are also eigenvectors of the shifted operators $D_{0}, D_{1}$ with the corresponding relations of the eigenvalues

$$
\frac{1}{\alpha}=\frac{1}{1-q}+\frac{w}{\sqrt{1-q}}, \quad \frac{1}{\beta}=\frac{1}{1-q}+\frac{v}{\sqrt{1-q}} .
$$

The choice of the boundary vectors to be deformed coherent states [19, 20] of the $q$-deformed oscillator $[20,21]$ set underlying the algebraic solution simplifies the procedure of obtaining all the relevant physical quantities. One has to merely normally order all the expressions monomials in $a, a^{+}$and use the coherent states properties. The representation has the proper $q=0$ limit which allows to obtain all the physical quantities of the totally asymmetric process as well. The deformed coherent state solution in the case of only incoming particle to the left and only outgoing particle to the right provides the most simple and convenient approach to a unified description of both the partially and the totally asymmetric processes.

The asymmetric exclusion process was studied and analyzed in relation to orthogonal polynomials technique. In the case of only injected particles at the left boundary and only removed at the right one the exact solution was related to the $q$-Hermite [16] and Al-Salam-Chihara polynomials [22]. In the general case of injected and removed particles at both boundaries with four nonzero boundary parameters the exact solution was shown to be related to the Askey-Wilson polynomials [23]. Our analysis aims to further stress the relation to the orthogonal polynomials. We put an emphasis on the important characteristic of the open asymmetric exclusion process that the bulk properties in the stationary state strongly depend on the boundary parameters. We find that the boundary operators generate the Askey-Wilson tridiagonal algebra whose irreducible modules are the Askey-Wilson polynomials. The boundary tridiagonal algebra reveals algebraic properties of the asymmetric open exclusion process which are deeply related to the Askey-Wilson polynomials and allow for the exact solution of the model.

\section{Symmetry of the open asymmetric exclusion process}

In the general case of incoming and outgoing particles at both boundaries there are four operators $\beta D_{1},-\delta D_{0},-\gamma D_{1}, \alpha D_{0}$ and one needs a pair of deformed oscillators to form two linear independent boundary operators acting on the dual vector spaces. To solve this problem within the matrix product ansatz we consider a particular form of a $q$-oscillator algebra, known in the literature as $C U_{q}(2)$ (or $(u, u), u>0$ ) algebra. It is generated by three elements with the defining commutation relations

$$
\left[N, A_{ \pm}\right]= \pm A_{ \pm}, \quad\left[A_{-}, A_{+}\right]=u q^{N}+u q^{-N}
$$

and a central element

$$
Q=A_{+} A_{-}+u \frac{q^{N}-q^{1-N}}{1-q} .
$$

This is an associative algebra and any invertible transformation of the generators is admissible. It can be presented in equivalent forms by means of the transformations

$$
\hat{A}_{+}=q^{N / 2} A_{+}, \quad \hat{A}_{-}=A_{-} q^{N / 2}
$$

with

$$
\left[\hat{A}_{-}, \hat{A}_{+}\right]_{q^{-1}}=u q^{2 N+1 / 2}+u q^{1 / 2}
$$


or

$$
\tilde{A}_{+}=q^{-N / 2} A_{+}, \quad \tilde{A}_{-}=A_{-} q^{-N / 2}
$$

with

$$
\left[\tilde{A}_{-}, \tilde{A}_{+}\right]_{q}=u q^{-1 / 2}+u q^{-2 N-1 / 2} .
$$

We consider now the two sets of operators

$$
a_{1}=q^{N / 2} A_{+}+q^{N}, \quad a_{1}^{+}=q^{-N / 2} A_{+}+\frac{1}{1-q} q^{-N}
$$

and

$$
a_{2}=A_{-} q^{N / 2}+\frac{1}{1-q^{-1}} q^{N}, \quad a_{2}^{+}=A_{-} q^{-N / 2}+q^{-N}
$$

obeying

$$
a_{1} a_{1}^{+}-q a_{1}^{+} a_{1}=1, \quad a_{2} a_{2}^{+}-q^{-1} a_{2}^{+} a_{2}=1 .
$$

This representation allows one to write the boundary operators in the equivalent form

$$
\begin{aligned}
\beta D_{1} & -\delta D_{0} \\
& =\frac{-x_{1} \beta}{1-q} q^{N / 2} A_{+}+x_{0} \delta q^{-1} A_{-} q^{N / 2}+\left(\frac{-x_{1} \beta}{(1-q)^{2}}+x_{0} \delta q^{-1}\right) q^{N}-\frac{x_{1} \beta}{1-q}-\frac{x_{0} \delta q^{-1}}{1-q^{-1}}, \\
\alpha D_{0} & -\gamma D_{1} \\
& =x_{0} \alpha q^{-N / 2} A_{+}-\frac{x_{1} \gamma q^{-1}}{1-q^{-1}} A_{-} q^{-N / 2}+\left(x_{0} \alpha-\frac{x_{1} \gamma q^{-1}}{\left(1-q^{-1}\right)^{2}}\right) q^{-N}+\frac{x_{0} \alpha}{1-q}+\frac{x_{1} \gamma q^{-1}}{1-q^{-1}} .
\end{aligned}
$$

We separate the shift parts from the boundary operators. Denoting the corresponding rest operator parts by $A$ and $A^{*}$ we write the left and right boundary operators in the form

$$
\beta D_{1}-\delta D_{0}=A-\frac{x_{1} \beta}{1-q}-\frac{x_{0} \delta q^{-1}}{1-q^{-1}}, \quad \alpha D_{0}-\gamma D_{1}=A^{*}+\frac{x_{0} \alpha}{1-q}+\frac{x_{1} \gamma q^{-1}}{1-q^{-1}} .
$$

Proposition 1. The operators $A$ and $A^{*}$ defined by

$$
A=\beta D_{1}-\delta D_{0}+\frac{x_{1} \beta}{1-q}+\frac{x_{0} \delta q^{-1}}{1-q^{-1}}, \quad A^{*}=\alpha D_{0}-\gamma D_{1}-\frac{x_{0} \alpha}{1-q}-\frac{x_{1} \gamma q^{-1}}{1-q^{-1}}
$$

and their q-commutator

$$
\left[A, A^{*}\right]_{q}=q^{1 / 2} A A^{*}-q^{-1 / 2} A^{*} A
$$

form a closed linear algebra

$$
\left[\left[A, A^{*}\right]_{q}, A\right]_{q}=-\rho A^{*}-\omega A-\eta, \quad\left[A^{*},\left[A, A^{*}\right]_{q}\right]_{q}=-\rho^{*} A-\omega A^{*}-\eta^{*},
$$

where the structure constants are given by

$$
\begin{aligned}
-\rho= & \beta \delta q^{-1} x_{1}^{2}\left(q^{1 / 2}+q^{-1 / 2}\right)^{2}, \quad-\rho^{*}=\alpha \gamma q^{-1} x_{0}^{2}\left(q^{1 / 2}+q^{-1 / 2}\right)^{2}, \\
-\omega= & \left(-x_{0} x_{1}\right) q^{-1}\left(\left(\beta+\delta\left(q^{1 / 2}-q^{-1 / 2}\right)^{2}\right)\left(\alpha\left(q^{1 / 2}-q^{-1 / 2}\right)^{2}+\gamma\right)\right. \\
& +\left(-x_{0} x_{1}\right) q^{-1}\left(q^{1 / 2}-q^{-1 / 2}\right)^{2} Q K,
\end{aligned}
$$




$$
\begin{aligned}
& \frac{-\eta}{q-q^{-1}}=x_{1} \beta x_{0} \delta q^{-1} Q\left(x_{0} \alpha+\frac{-x_{1} \gamma q^{-1}}{\left(1-q^{-1}\right)^{2}}\right)+K\left(\frac{-x_{1} \beta}{(1-q)^{2}}+x_{0} \delta q^{-1}\right), \\
& \frac{-\eta^{*}}{q-q^{-1}}=K\left(x_{0} \alpha+\frac{-x_{1} \gamma q^{-1}}{\left(1-q^{-1}\right)^{2}}\right)-x_{0} \alpha x_{1} \gamma q^{-1} Q\left(\frac{-x_{1} \beta}{(1-q)^{2}}+x_{0} \delta q^{-1}\right),
\end{aligned}
$$

where

$$
K=\left(-x_{o} x_{1}\right) q^{-1}\left(\alpha \delta-\frac{\beta \gamma}{\left(q^{1 / 2}-q^{-1 / 2}\right)^{2}}\right) .
$$

The proposition is straightforward to verify by using the representation for $A$ and $A^{*}$ on the RHS of formula (4.1). Relations (4.2) are the well known Askey-Wilson relations

$$
\begin{aligned}
& A^{2} A^{*}-\left(q+q^{-1}\right) A A^{*} A+A^{*} A^{2}=\rho A^{*}+\omega A+\eta, \\
& A^{* 2} A-\left(q+q^{-1}\right) A^{*} A A^{*}+A A^{* 2}=\rho^{*} A+\omega A^{*}+\eta^{*}
\end{aligned}
$$

for the shifted boundary operators $A, A^{*}$. The algebra (4.2) was first considered in the works of Zhedanov $[24,25]$ and recently discussed in a more general framework of a tridiagonal algebra [26, 27]. This is an associative algebra with a unit generated by a (tridiagonal) pair of operators $A$, $A^{*}$ and defining relations

$$
\begin{aligned}
& {\left[A, A^{2} A^{*}-\beta^{\prime} A A^{*} A+A^{*} A^{2}-\gamma^{\prime}\left(A A^{*}+A^{*} A\right)-\rho A^{*}\right]=0,} \\
& {\left[A^{*}, A^{* 2} A-\beta^{\prime} A^{*} A A^{*}+A A^{* 2}-\gamma^{\prime *}\left(A A^{*}+A^{*} A\right)-\rho^{*} A\right]=0 .}
\end{aligned}
$$

In the general case a tridiagonal pair is determined by the sequence of scalars $\beta^{\prime}, \gamma^{\prime}, \gamma^{*}, \rho, \rho^{*}$ from a field $K$. Note that we keep the conventional notations $\beta, \gamma, \rho$ for the scalars defining a tridiagonal pair in equation (4.6), however we use them with the index ' to avoid confusion with the boundary rates. Tridiagonal pairs have been classified according to the dependence on the scalars [26]. Examples are the $q$-Serre relations with $\beta^{\prime}=q+q^{-1}$ and $\gamma^{\prime}=\gamma^{\prime *}=\rho=\rho^{*}=0$

$$
\left[A, A^{2} A^{*}-\left(q+q^{-1}\right) A A^{*} A+A^{*} A^{2}\right]=0, \quad\left[A^{*}, A^{* 2} A-\left(q+q^{-1}\right) A^{*} A A^{*}+A A^{* 2}\right]=0
$$

and the Dolan-Grady relations [28] with $\beta^{\prime}=2, \gamma^{\prime}=\gamma^{*}=0, \rho=k^{2}, \rho^{*}=k^{* 2}$

$$
\left[A,\left[A,\left[A, A^{*}\right]\right]\right]=k^{2}\left[A, A^{*}\right], \quad\left[A^{*},\left[A^{*},\left[A^{*}, A\right]\right]\right]=k^{* 2}\left[A^{*}, A\right] .
$$

Tridiagonal pairs are determined up to an affine transformation

$$
A \rightarrow t A+c, \quad A^{*} \rightarrow t^{*} A^{*}+c^{*},
$$

where $t, t^{*}, c, c^{*}$ are some scalars. The affine transformation can be used to bring a tridiagonal pair in a canonical form with $\gamma^{\prime}=\gamma^{*}=0$.

The boundary operators of the asymmetric exclusion process obeying the Askey-Wilson algebra (4.2) form a tridiagonal pair with $\beta^{\prime}=q+q^{-1}, \gamma^{\prime}=\gamma^{*}=0$, and $\rho, \rho^{*}, \omega=\omega^{*}, \eta, \eta^{*}$ given by equations (4.3)-(4.5). The Askey-Wilson algebra possesses some important properties that allow to obtain its ladder representations, spectra, overlap functions. This fact immediately provides a solution to the boundary problem. We briefly touch the spectral problem following the argumentation of Zhedanov et al. (for details see [24, 25]). Let $f_{r}$ be an eigenvector of $A$ with eigenvalue $\lambda_{r}$

$$
A f_{r}=\lambda_{r} f_{r} .
$$

Then we can construct a new eigenstate

$$
f_{s}=\left(A g(A)+A^{*} h(A)+A_{0} k(A)\right) f_{r},
$$


where $A_{0}$ denotes the $q$-commutator $\left[A, A^{*}\right]_{q}$, and

$$
A f_{s}=\lambda_{s} f_{s} .
$$

It follows from the algebra that $f_{s}$ will also be an eigenvector of $A$, if for the new eigenvalue the quadratic relation holds

$$
\lambda_{r}^{2}+\lambda_{s}^{2}-\left(q+q^{-1}\right) \lambda_{r} \lambda_{s}-\rho=0 .
$$

This yields for each state $f_{r}$ two neighbouring states whose eigenvalues are the roots of the above quadratic equation. In this parametrization the operator $A$ is diagonal and the operator $A^{*}$ is tridiagonal

$$
A f_{r}=a_{r+1} f_{r+1}+b_{r} f_{r}+c_{r-1} f_{r-1} .
$$

The expressions for the spectrum and the matrix coefficient can be obtained explicitly, e.g. the spectrum reads (with $x_{1}^{2}=1$ )

$$
\lambda_{r}=q^{-1 / 2}(\beta \delta)^{1 / 2} \frac{q^{r}+q^{-r}}{q^{1 / 2}-q^{-1 / 2}} .
$$

The right boundary vector $|v\rangle$ can be thus related to a suitably chosen eigenstate of the shifted operator $A$. The algebra possesses a duality property. Due to the duality property the dual basis exists in which the operator $A^{*}$ is diagonal and the operator $A$ is tridiagonal. The left boundary vector $\langle w|$ will be related to an eigenstate of the shifted operator $A^{*}$ in the dual basis. We have

$$
A^{*} f_{p}^{*}=\lambda^{*} f_{p}^{*}, \quad A f_{s}^{*}=a_{s+1}^{*} f_{s+1}^{*}+b^{*} f_{s}^{*}+c_{s-1}^{*} f_{s-1}^{*} .
$$

The overlap function of the two basis $\langle s \mid r\rangle=\left\langle f_{s}^{*} \mid f_{r}\right\rangle$ is expressed in terms of the Askey-Wilson polynomials. To make the relation more transparent we rescale the generators

$$
A \rightarrow\left(q^{1 / 2}-q^{-1 / 2}\right) \frac{1}{\beta} \sqrt{\frac{\gamma}{\alpha}} A, \quad A^{*} \rightarrow\left(q^{1 / 2}-q^{-1 / 2}\right) \frac{q^{1 / 2}}{\sqrt{\alpha \gamma}} A^{*} .
$$

The tridiagonal relations for the transformed operators read

$$
\begin{aligned}
& {\left[A, A^{2} A^{*}-\left(q+q^{-1}\right) A A^{*} A+A^{*} A^{2}+a b c d q^{-1}\left(q-q^{-1}\right)^{2} A^{*}\right]=0,} \\
& {\left[A^{*}, A^{* 2} A-\left(q+q^{-1}\right) A^{*} A A^{*}+A A^{* 2}+\left(q-q^{-1}\right)^{2} A\right]=0,}
\end{aligned}
$$

where $a b c d=\frac{\gamma}{\alpha} \frac{\delta}{\beta}$. Let $p_{n}=p_{n}(x ; a, b, c, d)$ denote the $n$th Askey-Wilson polynomial [29] depending on four parameters $a, b, c, d$

$$
p_{n}(x ; a, b, c, d)={ }_{4} \Phi_{3}\left(\begin{array}{c}
q^{-n}, a b c d q^{n-1}, a y, a y^{-1} \\
a b, a c, a d
\end{array} \mid q ; q\right)
$$

with $p_{0}=1, x=y+y^{-1}$ and $0<q<1$. On the RHS of equation (4.7) we use the standard notations for the basic hypergeometric series given in [30]. Then it can be proved (see [27] for details) that $p_{n}$ is an eigenstate of the operator $A$

$$
A p_{n}=\left(q^{n}+q^{-1} a b c d q^{-n}\right) p_{n}, \quad n=0,1,2, \ldots
$$

The operator $A^{*} p_{n}=x p_{n}$ is tridiagonal and its matrix elements in the basis

$$
\left(p_{0}(x), p_{1}(x), p_{2}(x), \ldots\right)
$$


are obtained from

$$
x p_{n}=b_{n} p_{n+1}+a_{n} p_{n}+c_{n} p_{n-1}, \quad p_{-1}=0
$$

which is the three term recurrence relation [29] of the Askey-Wilson polynomials. Let $\mathcal{A}$ denote the tridiagonal matrix

$$
\mathcal{A}=\left(\begin{array}{cccc}
a_{0} & c_{1} & & \\
b_{0} & a_{1} & c_{2} & \\
& b_{1} & a_{2} & . \\
& & \cdot & .
\end{array}\right)
$$

representing the operator $A^{*}$ in the basis (4.8). The spectrum of the boundary operators is obtained by the correspondingly rescaled shifts. We will summarize the results. In the representation $\pi$ with basis

$$
\left(p_{0}(x), p_{1}(x), p_{2}(x), \ldots\right)^{t}
$$

the operator $D_{1}-\frac{\delta}{\beta} D_{0}$ is diagonal with diagonal eigenvalues

$$
\lambda_{n}=\frac{1}{1-q}\left(b q^{-n}+d q^{n}+1+b d\right)
$$

and the operator $D_{0}-\frac{\gamma}{\alpha} D_{1}$ is tridiagonal

$$
\pi\left(D_{0}-\frac{\gamma}{\alpha} D_{1}\right)=\frac{1}{1-q}\left(b \mathcal{A}^{t}+1+a c\right) .
$$

In the dual representation $\pi^{*}$ with respect to the basis (4.8) the operator $D_{0}-\frac{\gamma}{\alpha} D_{1}$ is diagonal with eigenvalues

$$
\lambda_{n}^{*}=\frac{1}{1-q}\left(a q^{-n}+c q^{n}+1+a c\right)
$$

and the operator $D_{1}-\frac{\delta}{\beta} D_{0}$ is tridiagonal

$$
\pi\left(D_{1}-\frac{\delta}{\beta} D_{0}\right)=\frac{1}{1-q}(a \mathcal{A}+1+b d)
$$

The Askey-Wilson parameters $a, b, c, d$ on the RHS of equations (4.9), (4.10) and (4.11), (4.12) depend on the four boundary rates $\alpha, \beta, \gamma, \delta$.

The left and right boundary vectors can be chosen (up to a normalization) as

$$
\left\langle w\left|=\left(p_{0}, 0,0, \ldots\right), \quad\right| v\right\rangle=\left(p_{0}, 0,0, \ldots\right)^{t} .
$$

Then, the solutions to the eigenvalue equations determine uniquely, in the considered representation of the boundary operators, the relation of the boundary rates to the four Askey-Wilson parameters. The explicit expressions read

$$
a=\kappa_{+}^{*}, \quad b=\kappa_{+}, \quad c=\kappa_{-}^{*}, \quad d=\kappa_{-},
$$

where

$$
\kappa_{ \pm}=\frac{-(\beta+\delta-(1-q)) \pm \sqrt{(\beta+\delta-(1-q))^{2}-4 \beta \delta}}{2 \beta},
$$




$$
\kappa_{ \pm}^{*}=\frac{-(\alpha+\gamma-(1-q)) \pm \sqrt{(\alpha+\gamma-(1-q))^{2}-4 \alpha \gamma}}{2 \alpha} .
$$

We note the different relations compared to the corresponding ones in [23]. The boundary symmetry has rich algebraic properties and depending on its representation one has various identifications of the boundary rates dependence with the parameters of the Askey-Wilson polynomials.

We thus conclude: The quadratic algebra of the bulk diffusion process define a quantum plane with the $S U_{q}(2)$ action as its symmetry. The boundary processes amount to the presence of linear terms in the quadratic algebra and lead to a reduction of the bulk symmetry. The Askey-Wilson algebra arises as a symmetry of the boundary problem. The boundary vectors are expressed in terms of the Askey-Wilson polynomials whose four parameters are related to the four boundary probability rates.

\section{Acknowledgments}

The author would like to thank the organizers for the invitation to participate the O'Raifeartaigh symposium and for the warm and friendly atmosphere during the stay in Budapest.

\section{References}

[1] Schuetz G.M., Phase transitions and critical phenomena, Vol. 19, Academic Press, London, 2000.

[2] Schreckenberg M., Schadschneider A., Nagel K., Ito N., Discrete stochastic models for traffic flow, Phys. Rev. E 51 (1995), 2939-2949, cond-mat/9412045.

[3] Macdonald J.T., Gibbs J.H., Pipkin A.C., Kinetics of biopolymerization on nucleic acid templates, Biopolymers 6 (1968), 1-25.

[4] Krug J., Spohn H., Kinetic roughening of growing surfaces, in Solids Far from Equilibrium, Editor C. Godreche, Cambridge University Press, Cambridge, 1991, 412-525.

[5] Sandow S., Schuetz G.M., On $U_{q}(S U(2))$-symmetric driven diffusion, Europhys. Lett. 26 (1994), 7-13, cond-mat/9307027.

[6] Derrida B., Evans M.R., Hakim V., Pasquier V., Exact solution of a 1D asymmetric exclusion model using a matrix formulation, J. Phys. A: Math. Gen. 26 (1993), 1493-1517.

[7] Derrida B., An exactly soluble non-equilibrium system: the asymmetric simple exclusion process, Phys. Rep. 301 (1998), 65-83 (and references therein).

[8] Schuetz G.M., Domany E., Phase transitions in an exactly soluble one-dimensional asymmetric exclusion model, J. Stat. Phys. 72 (1993), 277-296, cond-mat/9303038.

[9] Krug J., Boundary-induced phase transitions in driven diffusive systems, Phys. Rev. Lett. 67 (1991), 18821885.

[10] Isaev A., Pyatov P., Rittenberg V., Diffusion algebras, J. Phys. A: Math. Gen. 34 (2001), 5815-5834, cond-mat/0103603.

[11] Aneva B., The noncommutative space of stochastic diffusion systems, J. Phys. A: Math. Gen. 35 (2002), 859-877.

[12] Essler F.H.L., Rittenberg V., Representations of the quadratic algebra and partially asymmetric diffusion with open boundaries, J. Phys. A: Math. Gen. 29 (1996), 3375-3407, cond-mat/9506131.

[13] Arndt P.F., Heinzel T., Rittenberg V., Stochastic models on a ring and quadratic algebras. The three-species diffusion problem, J. Phys. A: Math. Gen. 31 (1998), 833-843, cond-mat/9703182.

[14] Fairlie D., Zachos C., Multiparameter associative generalizations of canonical commutation relations and quantized planes, Phys. Lett. B 256 (1991), 43-49.

[15] Wess J., Zumino B., Covariant differential calculus on the quantum hyperplane, Nucl. Phys. B, Proc. Suppl. 18 (1990), 302-312.

[16] Blythe R.A., Evans M.R., Colaiori F., Essler F.H.L., Exact solution of a partially asymmetric exclusion model using a deformed oscillator algebra, J. Phys. A: Math. Gen. 33 (2000), 2313-2332. 
[17] Sandow S., Partially asymmetric exclusion process with open boundaries, Phys. Rev. E 50 (1994), 26602667, cond-mat/9405073.

[18] Stinchcombe R.B., Schuetz G.M., Application of operator algebras to stochastic dynamics and the Heisenberg chain, Phys. Rev. Lett. 75 (1995), 140-143.

[19] Arik M., Coon D.D., Hilbert spaces of analytic functions and generalized coherent states, J. Math. Phys. 17 (1976), 524-527.

[20] Kulish P.P., Damaskinsky E.V., On the $q$ oscillator and the quantum algebra $s u_{q}(1,1)$, J. Phys. A: Math. Gen. 23 (1990), L415-L419.

[21] Chaichian M., Kulish P.P., Quantum Lie superalgebras and q-oscillators, Phys. Lett. B 234 (1990), 72-80.

[22] Sasamoto T., One-dimensional partially asymmetric simple exclusion process with open boundaries: orthogonal polynomials approach, J. Phys. A: Math. Gen. 32 (1999), 7109-7131.

[23] Uchiyama M., Sasamoto T., Wadati M., Asymmetric simple exclusion process with open boundaries and Askey-Wilson polynomials, J. Phys. A: Math. Gen. 37 (2004), 4985-5002, cond-mat/0312457.

[24] Zhedanov A.S., Hidden symmetry of Askey-Wilson polynomials, Teoret. Mat. Fiz. 89 (1991), $190-204$.

[25] Granovskii Y.A., Lutsenko I.M., Zhedanov A.S., Mutual integrability, quadratic algebras and dynamical symmetry, Ann. Physics 217 (1992), 1-20.

[26] Terwilliger P., An algebraic approach to the Askey scheme of orthogonal polynomials, Lecture Notes in Mathematics, Vol. 1883, Editors F. Marcellan and W.V. Assche, Springer, Berlin, 2006, 225-330.

[27] Terwilliger P., Two relations that generalize the $q$-Serre relations and the Dolan-Grady relations, Proceedings of the Nagoya 1999 International Workshop on Physics and Combinatorics, Editors A.N. Kirillov, A. Tsuchiya and H. Umemura, World Sci. Pub., River Edge, NJ, 2001, 377-398, math.QA/0307016.

[28] Dolan L., Grady M., Conserved charges from self-duality, Phys. Rev. D25 (1982), 1587-1604.

[29] Askey R.A., Wilson J.A., Some basic hypergeometric orthogonal polynomials that generalize Jacobi polynomials, Mem. Amer. Math. Soc. (1985), no. 319, 55 pages.

[30] Gasper G., Rahman M., Basic hypergeometric series, Encyclopedia of Mathematics and Its Applications, Vol. 35, Cambridge University Press, Cambridge, 1990. 